\title{
Reduced regional flow in the left ventricle after anterior acute myocardial infarction: a case control study using 4D flow MRI
}

\author{
Philip A. Corrado ${ }^{1 *}$ (D, Jacob A. Macdonald ${ }^{1}$, Christopher J. François ${ }^{2}$, Niti R. Aggarwal ${ }^{3}$, Jonathan W. Weinsaft ${ }^{4}$ and
} Oliver Wieben ${ }^{5}$

\begin{abstract}
Background: Acute myocardial infarction (AMI) alters left ventricular (LV) hemodynamics, resulting in decreased global LV ejection fraction and global LV kinetic energy. We hypothesize that anterior AMI effects localized alterations in LV flow and developed a regional approach to analyze these local changes with 4D flow MRI.

Methods: 4D flow cardiac magnetic resonance (CMR) data was compared between 12 anterior AMI patients (11 males; $66 \pm 12$ yo; prospectively acquired in 2016-2017) and 19 healthy volunteers (10 males; $40 \pm 16 y$;

retrospective from 2010 to 2011 study). The LV cavity was contoured on short axis cine steady-state free procession CMR and partitioned into three regions: base, mid-ventricle, and apex. 4D flow data was registered to the short axis segmentation. Peak systolic and diastolic through-plane flows were compared region-by-region between groups using linear models of flow with age, sex, and heart rate as covariates.

Results: Peak systolic flow was reduced in anterior AMI subjects compared to controls in the LV mid-ventricle (fitted reduction $=3.9 \mathrm{~L} / \mathrm{min} ; P=0.01$ ) and apex (fitted reduction $=1.4 \mathrm{~L} / \mathrm{min} ; P=0.02$ ). Peak diastolic flow was also lower in anterior AMI subjects compared to controls in the apex (fitted reduction $=2.4 \mathrm{~L} / \mathrm{min} ; \mathrm{P}=0.01$ ).

Conclusions: A regional method to analyze 4D LV flow data was applied in anterior AMI patients and controls. Anterior AMI patients had reduced regional flow relative to controls.
\end{abstract}

Keywords: Phase contrast, 4D flow, Myocardial infarction, Left ventricle

\section{Background}

Acute myocardial infarction (AMI) is widespread [1] and has high mortality and morbidity [2]. AMI alters left ventricular (LV) hemodynamics, resulting in increased left ventricular volumes and decreased left ventricular ejection fraction (LVEF) - both powerful prognostic indicators post-AMI [3]. A common complication of AMI is left ventricular thrombus (LVT) - a causal substrate for stroke [4]. In a prospective study of 201 AMI patients, LVT were identified in $8 \%$ of all subjects and in $15 \%$ of those with anterior infarctions using cardiac magnetic resonance (CMR) within 30 days of infarction, with all thrombi located in the LV apex [5]. The

\footnotetext{
*Correspondence: pcorrado2@wisc.edu

${ }^{1}$ Department of Medical Physics, University of Wisconsin-Madison, 1111

Highland Ave, Madison, WI 53705, USA

Full list of author information is available at the end of the article
}

pathogenesis of LVT is caused by a combination of blood stasis, endothelial injury and hypercoagulability, often referred to as Virchow's triad [6].

Velocity-sensitive imaging offers the opportunity to investigate blood stasis in the post-AMI left ventricle, shedding light on the mechanisms behind high rates of LVT. Several studies to date have investigated LV hemodynamics after myocardial infarction (MI). In a prospective study using two-dimensional (2D) Doppler echocardiography in 104 AMI patients, Dantzig et al. found that abnormal flow (defined as the presence of rotating flow in the apex and/or vortex ring formation) was independently predictive of LVT [7]. However, Doppler echocardiography is limited in spatial coverage (imaging planes are restricted by patient anatomy) and non-specific velocity direction encoding. Another study, using time-resolved, three-dimensional (3D) phase contrast 
MRI with 3-directional velocity encoding ("4D flow MRI" $[8,9])$ in 48 patients with acute or chronic myocardial infarction (MI), found reduced LV kinetic energy in MI patients compared to age/sex-matched controls [10].

While these studies demonstrate that MI alters LV hemodynamics, 4D flow MRI has not yet been applied to look specifically at regional flow differences in anterior AMI patients (the population most relevant to apical LVT risk). Since post-MI LVT typically localizes in the LV apex, we hypothesize that the apex will be most acutely affected by post-anterior-AMI flow reductions. The purpose of this study was to investigate differences in regional intraventricular flow in the LV base, midventricle, and apex between anterior AMI patients and healthy controls using 4D flow MRI.

\section{Methods}

\section{Study population}

Anterior AMI patients were scanned prospectively with 4D flow MRI as an adjunct to their clinical CMR exams performed in 2016-2017. These patients were compared against historical controls from a previously reported, 2010-2011 study [11]. This study was approved by the Institutional Review Board and was compliant with the Health Insurance Portability and Accountability Act. Written informed consent was obtained from all subjects. Inclusion criteria for anterior AMI patients were hospitalization and revascularization for AMI with the left anterior descending artery or left main coronary artery identified as the culprit vessel by coronary angiography, and a clinically ordered CMR exam. Exclusion criteria were contraindications to MRI or gadoliniumbased contrast agents. Twelve patients with anterior AMI were recruited. Exclusion criteria for control subjects were standard contraindications to MRI and to gadolinium-based contrast agents, high cardiovascular risk factors (body mass index $>30$, history of smoking, diabetes, or hypertension), and drugs affecting cardiovascular function. Data from 19 control subjects were included in the analysis, for a total of 31 scans analyzed.

\section{MRI acquisition}

CMR examinations in controls were performed in a 3.0 $\mathrm{T}$ scanner (MR750, GE Healthcare, Waukesha, WI). CMR examinations in AMI subjects were acquired on $1.5 \mathrm{~T}$ (MR450w or HDxt, GE Healthcare, Waukesha, WI; $N=9$ ) and 3.0 T (MR750 or MR750w, GE Healthcare, Waukesha, WI; $N=3$ ) scanners. The choice of field strength was based on the clinical availability of the scanners. The CMR protocol included a short-axis bSSFP cine acquisition to segment the LV cavity \& compute LV strain, 4D flow imaging for velocity mapping, and short-axis late gadolinium enhancement (LGE; only in AMI patents) imaging to measure infarction size. 4D flow data was acquired with PC VIPR, a threedimensional radially-undersampled, three-directionally velocity encoded technique $[12,13]$, with a scan duration of 9-14 min including respiratory gating efficiency. Each set of 4D flow data was reconstructed in two ways: a high-resolution image set was reconstructed using a gridding technique and used to measure through-plane flow and intraventricular KE, and a low-resolution, lownoise image set was reconstructed using compressed sensing with a spatial-wavelet-transform L1-norm penalty $(\lambda=0.01)$ in order to separate LV flow into different compartments by tracking flow pathlines. This separate reconstruction was used for pathline tracking because this method is sensitive to noise due to compounding errors in pathline integration. Intravenous contrast was administered to all subjects prior to 4D flow imaging. AMI patients received $0.15 \mathrm{mmol} / \mathrm{kg}$ of gadobenate dimeglumine (Multihance; Bracco, Milan, IT) and controls received $0.03 \mathrm{mmol} / \mathrm{kg}$ of gadofosveset trisodium (Ablavar; Lantheus, Billerica, MA, USA). Table 1 shows the MRI acquisition parameters.

\section{Image analysis}

The LV cavity was segmented at each time frame on short-axis bSSFP images (including outflow tract, excluding papillary muscles) using the software Segment (Medviso, http://segment.heiberg.se; v2.0 R5399) [14]. LV end diastolic volume (EDV), end systolic volume (ESV), stroke volume (SV), and ejection fraction, were calculated from the endocardial borders. Global longitudinal strain (GLS) was computed from long-axis bSSFP images, and regional radial and circumferential strain were computed from short-axis bSSFP images using one slice each in the base, mid-ventricle, and apex in Segment using feature tracking [15].

4D flow data background phase errors were corrected by fitting a 3rd order polynomial to static tissue phase. Subsequently, 4D flow data was registered to the shortaxis bSSFP dataset using the Advanced Normalization Toolkit [16, 17]. Proper registration was confirmed by overlaying the images in ITK-SNAP [18]. Each slice in the LV segmentation was assigned to one of three equallength segments divided along the LV long axis: base, mid-ventricle, and apex (Fig. 1). Through-plane flow was computed for each slice and time point by multiplying the average through-plane velocity component in the LV with the cross-sectional area of the LV. Through-plane flow in each LV region was computed as the average through-plane flow for each slice in that region. Peak systolic and diastolic flows were defined for each region as maximum positive and negative flows, with the positive through-plane direction running from the apex to the base. 
Table $1 \mathrm{MRI}$ acquisition parameters

\begin{tabular}{|c|c|c|c|}
\hline Parameter & 2D Short-Axis bSSFP & 4D flow (PC VIPR) & 2D LGE Scar Imaging \\
\hline ECG type & prospectively gated & retrospectively gated & triggered (diastolic phase) \\
\hline Cine Frames & 20 & 20 & - \\
\hline Respiratory Motion Strategy & breath holding & free-breathing & breath holding \\
\hline Repetition Time (ms) & $3.1-4.1$ & $5.8-8.4$ & $4.0-4.8$ \\
\hline Echo Time (ms) & $1.1-1.2$ & $2.0-2.5$ & $1.4-2.2$ \\
\hline Flip Angle (degrees) & 45 & $8-12$ & $20-45$ \\
\hline Inversion Time $(\mu s)$ & - & - & $183-400$ \\
\hline Field of View $\left(\mathrm{cm}^{2}\right)$ & $39 \times 39$ & - & $35 \times 35$ \\
\hline Slice Thickness (mm) & 8 & - & 8 \\
\hline Imaging Volume $\left(\mathrm{cm}^{3}\right)$ & - & $32 \times 32 \times 20$ & - \\
\hline Acquired Spatial Resolution (mm) & $1.74 \times 1.74$ & $1.25 \times 1.25 \times 1.25$ & $2.03 \times 2.03$ \\
\hline Reconstructed Spatial Resolution (mm) & $1.25 \times 1.25$ & $\begin{array}{l}\text { High resolution reconstruction: } \\
1.25 \times 1.25 \times 1.25 \\
\text { Low resolution reconstruction: } \\
2.5 \times 2.5 \times 2.5\end{array}$ & $1.37 \times 1.37$ \\
\hline Velocity Encoding (cm/s) & - & $100-150$ & - \\
\hline
\end{tabular}

Note-Reported values are the ranges (minima and maxima) for each parameter. Parameters were tailored to each individual's anatomy and the field strength used. Two sets of images were reconstructed for each 4D flow acquisition: a high-resolution image set used to compute flow and ventricular KE, and a lowresolution image set used to separate LV flow components by tracking pathlines

Average KE was computed by summing the KE contributions for all voxels in the LV and averaging over time:

$$
\begin{aligned}
K E_{\text {avg }} & =\frac{1}{N_{t}} \sum_{\text {time }} \sum_{\text {voxels }} K E_{\text {vox }} \\
& =\frac{1}{N_{t}} \sum_{\text {time }} \sum_{\text {voxels }} \frac{1}{2} \rho_{\text {blood }} V_{\text {vox }} v_{\text {vox }}^{2}
\end{aligned}
$$

Where $\mathrm{N}_{\mathrm{t}}$ is the number of cardiac time frames, $\rho_{\text {blood }}$ is the density of blood $\left(1.06 \mathrm{~g} / \mathrm{cm}^{3}\right), \mathrm{V}_{\mathrm{vox}}$ is the voxel volume, and $\mathrm{v}_{\mathrm{vox}}$ is the velocity magnitude. $\mathrm{KE}_{\mathrm{avg}}$ was then indexed to EDV ( $\left.\mathrm{KEi}_{\mathrm{EDV}}\right)$ as in Garg et al. [10]. KE was not computed on a regional scale because the squared velocity term results in a measurement dominated by high-velocity voxels, which is therefore highly sensitive to noise in slow-flow regions (such as the LV apex) with a full-ventricle high velocity encoding imaging approach.

The distribution of different LV flow components was determined in all subjects using the method of Eriksson et al. [19]. Blood pathlines were emitted from the LV blood volume and traced forwards and backwards in time from end diastole until end systole, thus including the entire cardiac cycle. Pathlines were computed by integrating the velocity field using a 4th order Range-
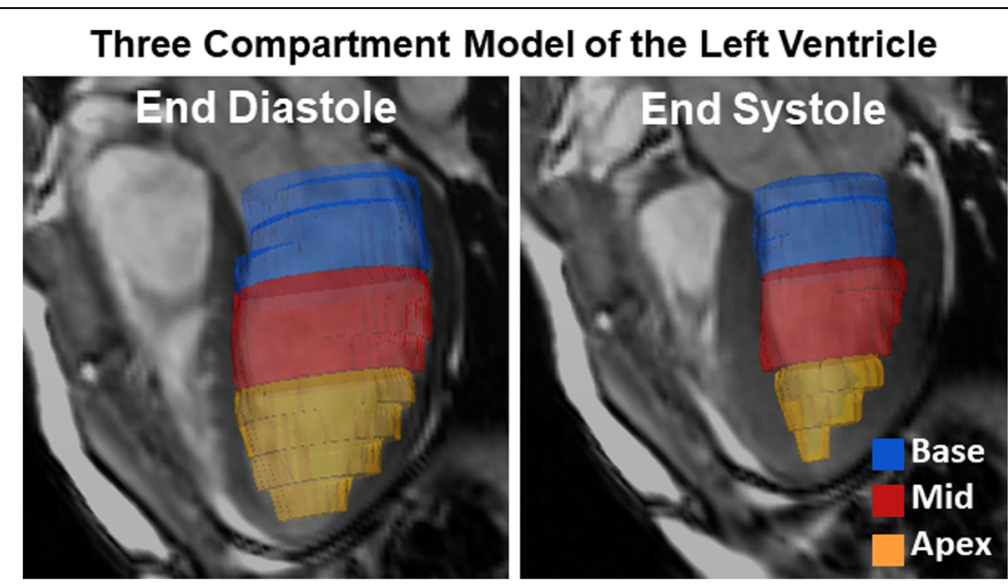

Fig. $13 D$ visualization of time-resolved LV segmentation produced from cine short-axis bSSFP images, displayed at end diastole (left) and end systole (right). The LV cavity was subdivided into three regions along the LV long axis for regional flow analysis: base (blue), mid-ventricle (red), and apex (orange). A co-registered long-axis bSSFP image slice is overlaid for viewing convenience. These visualizations were generated using Ensight 10.0 (Ansys, Canonsburg, PA) 
Kutta numerical integration through time. Pathline location was used to separate the pathlines into four different components of flow: Direct Flow (blood that enters the LV during diastole and leaves the LV during systole in the analyzed heartbeat), Retained Inflow (blood that enters the LV during diastole but does not leave during systole in the analyzed heartbeat), Delayed Ejection Flow (blood that starts and resides inside the LV during diastole and leaves during systole), and Residual Volume (blood that resides within the LV for at least two cardiac cycles). Pathlines passing through the ventricle wall (either entering or leaving the LV through the midventricle or apical regions) were excluded from analysis. The fraction of EDV containing pathlines from each compartment was computed for all subjects.

All flow computations were performed in Matlab (R2018a, The Mathworks Inc., Natick, Massachusetts, USA). All bSSFP and 4D flow images were analyzed by PAC (3 years of CMR analysis experience). Kim's method [20] was used on LGE images to compute infarct size as follows: each segment in the 17-segment AHA myocardial model was scored for infarction transmurality using a 5 -point scale $(0=$ no infarction, $1=0$ $25 \%, 2=25-50 \%, 3=50-75 \%, 4=>75 \%$ transmurality), the scores were averaged, and the result was divided by four. The images were scored by consensus of two radiologists with expertise in cardiothoracic imaging (reader 1, 17 years of experience; reader 2, 5 years of experience). Disagreements were handled by consulting a third radiologist. The LGE readers were blinded to the results of the flow analysis and vice-versa.

\section{Statistical analysis}

Demographic and traditional CMR measures (LV function and strain) are presented as mean \pm standard deviation and were compared between AMI patients and controls using independent sample t-tests. The proportion of male subjects was compared between groups using a chi-squared test. The $11 \mathrm{LV}$ flow parameters (peak systolic and diastolic flow in each region, global $\mathrm{KEi}_{\mathrm{EDV}}$, and the percentage of LV flow in each of the 4 compartments) are not assumed to be normally distributed and are presented as median \pm inter-quartile range (IQR). Multivariable linear regression was used to test if there were differences in the $11 \mathrm{LV}$ flow parameters between anterior AMI patients and controls, while adjusting for age, sex and heart rate differences between the groups as covariates. For each LV flow parameter, a multivariable linear regression model was fitted with LV flow parameter as outcome, group of subjects (AMI patient or control) as predictor variable, and age, sex, and heart rate as covariates in the model. Model diagnostics were performed and no serious violations of the assumptions of the linear models were found. Regional
LV through-plane flow parameters were correlated with heart rate and traditional CMR measures (infarct size, SV, $\mathrm{CO}, \mathrm{EF}, \mathrm{EDV}$, GLS, and regional radial and circumferential strain) using Spearman's rank correlation test. Regional through-plane flow and regional strain were compared on a region-by-region basis (i.e. flow in the LV base was compared with strain in the LV base). Multivariable regression modeling was performed in $R$ version 3.5.2. All other analysis was performed in MATLAB. A significance level of 0.05 was used for all tests.

\section{Results \\ Study population}

Ten of the 12 anterior AMI subjects (83\%) had STsegment elevation. The mean interval between AMI and CMR was 3.7 days with a range of 1-13 days. Peak measured troponin-I levels in AMI subjects had a mean of $119.1 \mathrm{ng} / \mathrm{mL}$ and a range of $0.08-309.9 \mathrm{ng} / \mathrm{mL}$. The mean percentage of myocardium infarcted was $26 \%$ with a range of $4-53 \%$. Three AMI subjects (25\%) had a left ventricular thrombus present at the time of imaging. Table 2 shows demographics and traditional CMR measures for healthy controls and AMI subjects.

\section{LV flow measurements}

For all subjects analyzed, 4D flow data was successfully registered to short-axis cine images for through-plane velocity mapping. Figure 2 shows examples of throughplane velocity and KE mapping at peak systole in a representative control subject and in a representative subject with an anterior AMI. Compared to the control subject, the anterior AMI subject has lower throughplane velocities in all LV regions. In both subjects, the KE map is dominated by regions near the left ventricular outflow tract. Figure 3 shows average through-plane flow-time curves in each region for anterior AMI patients and controls. Figure 4 shows representative visualizations of flow compartment analysis in a control $(\mathrm{a}, \mathrm{b})$ and AMI subject (d, e). The AMI subject had a lower fraction of pathlines in the direct flow compartment and a higher fraction of pathlines in the residual volume compartment. Subpanels c) and f) show group-average pie charts of the distribution of flow among compartments. Among all subjects, the median number of pathlines passing through the ventricular wall (and therefore discarded) was $24 \%$. Table 3 shows the average $12 \mathrm{LV}$ flow parameters in controls and anterior AMI patients, along with $P$-values for the comparison. Compared to controls, anterior AMI subjects had significantly lower through-plane flow in the mid-ventricle at peak systole and in the apex at peak systole and diastole. There were no significant differences in $\mathrm{KEi}_{\mathrm{EDV}}$ measures between groups. Compared to controls, anterior AMI subjects had significantly less direct flow and significantly more 
Table 2 Demographics, LV function, and LV strain measures in all subjects

\begin{tabular}{|c|c|c|c|}
\hline & Controls $(n=19)$ & Anterior AMI Patients $(n=12)$ & $P$-value \\
\hline Sex (male, n) & 10 & 11 & 0.02 \\
\hline Age (years) & $40 \pm 16$ & $66 \pm 12$ & $2 \mathrm{E}-05$ \\
\hline Weight (kg) & $76 \pm 11$ & $86 \pm 25$ & 0.23 \\
\hline Heart rate (bpm) & $60 \pm 10$ & $79 \pm 15$ & 0.002 \\
\hline Stroke volume $(\mathrm{mL})$ & $95 \pm 16$ & $65 \pm 21$ & $5 \mathrm{E}-04$ \\
\hline Cardiac output (L/min) & $5.6 \pm 0.9$ & $5 \pm 1.1$ & 0.10 \\
\hline End diastolic volume (mL) & $141 \pm 24$ & $149 \pm 32$ & 0.46 \\
\hline Ejection fraction (\%) & $68 \% \pm 6 \%$ & $44 \% \pm 10 \%$ & $2 \mathrm{E}-06$ \\
\hline \multicolumn{4}{|l|}{ Radial strain (\%) } \\
\hline Base & $31 \% \pm 9 \%$ & $33 \% \pm 14 \%$ & 0.73 \\
\hline Mid-ventricle & $42 \% \pm 9 \%$ & $28 \% \pm 18 \%$ & 0.02 \\
\hline Apex & $36 \% \pm 18 \%$ & $14 \% \pm 20 \%$ & 0.01 \\
\hline \multicolumn{4}{|l|}{ Circumferential strain (\%) } \\
\hline Base & $-18 \% \pm 2 \%$ & $-12 \% \pm 4 \%$ & $1 \mathrm{E}-04$ \\
\hline Mid-ventricle & $-19 \% \pm 3 \%$ & $-10 \% \pm 5 \%$ & $2 \mathrm{E}-05$ \\
\hline Apex & $-21 \% \pm 4 \%$ & $-6 \% \pm 5 \%$ & $5 \mathrm{E}-08$ \\
\hline Longitudinal strain (\%) & $-16 \% \pm 2 \%$ & $-7 \% \pm 3 \%$ & $1 \mathrm{E}-07$ \\
\hline
\end{tabular}

Note-Reported values are mean \pm 1 standard deviation. $P$-values were computed using two-sample t-tests. Boldface signifies $P<0.05$

retained inflow, delayed ejection flow, and residual volume.

\section{Correlation of LV flow parameters with infarct size and traditional CMR measures}

Spearman's rank correlation coefficient was used to determine the correlation of heart rate (HR) and traditional CMR metrics (infarct size, LV SV, LV CO, LV EF, LV EDV, GLS, and regional radial and circumferential strain) with through-plane flows in each region at peak systole and diastole. Table 4 shows the $P$-values for the correlation coefficient, with the coefficient in parentheses for significant correlations. Peak basal systolic through-plane flow was negatively correlated with HR and peak basal circumferential strain and positively correlated with SV and EF. Peak mid-ventricular throughplane flow was also positively correlated with EF. There were no significant correlations with through-plane flow in the apex or with any diastolic flow measures.

\section{Discussion}

The focus of this study was to apply 4D flow MRI to compare regional and global LV flow between anterior AMI patients and controls. To do so, through-plane flows in the LV base, mid-ventricle, and apex, as well as global $\mathrm{KEi}_{\mathrm{EDV}}$ and the fraction of LV flow in each of the 4 flow compartments, were measured in 12 patients and 19 controls. AMI patients had reduced through-plane flow in the apex at peak systole and diastole, and in the mid-ventricle at peak systole compared to controls, even after correcting for age, sex, and heart rate differences between the groups. While the other flow measurements were also lower in patients than in controls, the differences were not significant after correcting for age, sex, and heart rate differences. This finding supports the notion of stasis in the LV apex of anterior AMI subjects contributing to the elevated rates of LVT experienced by this group. The implication of this finding is that $4 \mathrm{D}$ flow MRI of the LV apex may be a valuable tool in larger studies on post-AMI LVT risk assessment. While the more traditional hemodynamic metric, LVEF, was also lower in patients than in controls, this measure does not offer insights into the location of flow impairment. This study did not find the reduction in $\mathrm{KEi}_{\mathrm{EDV}}$ that Garg et al. found in a cohort of $48 \mathrm{MI}$ subjects [10]. This is likely caused by the smaller sample size of this study and the use of different scanners for different subjects, which we elaborate on in the limitations paragraph below. Flow compartment analysis revealed a marked shift away from blood entering and leaving the LV in one heartbeat (direct flow) towards blood starting and residing in the LV for $>=2$ heartbeats (residual volume) in anterior AMI patients. This increase in residual volume is consistent with the decreased through-plane flow in the apex (since residual flow pathlines are typically located in or near the apex) and with the notion of increased stasis post MI. Our control data have similar flow rates to those reported in 1995 [21] and similar KE values to those reported in 2015 [22] and 2016 [23] in healthy controls. Our control data however displays a greater fraction of 


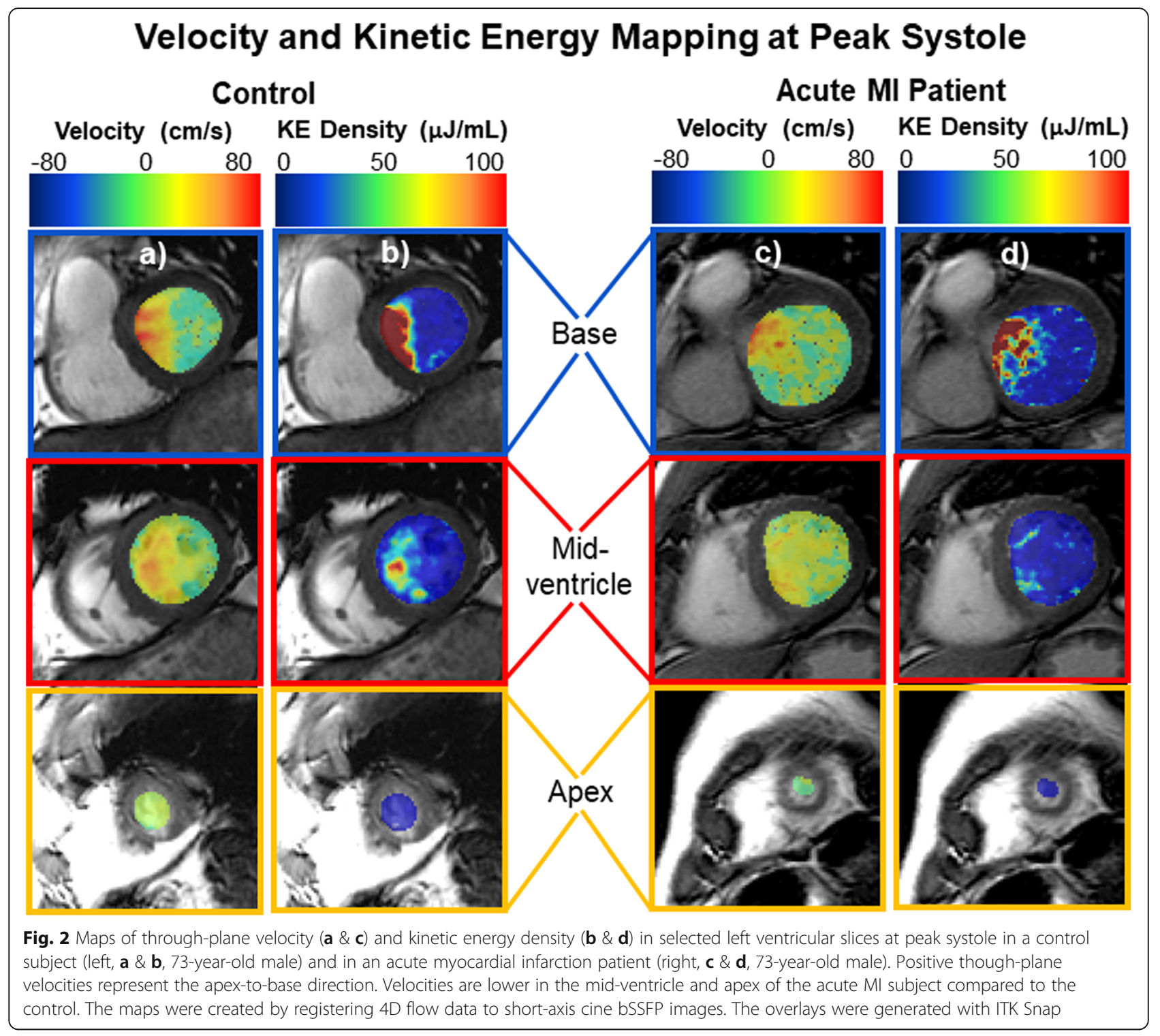

flow in the direct flow compartment and less in the residual volume compartment than Eriksson et al. found in 2010 [19] and 2013 [24]. We attribute this to differences in acquisition such as k-space trajectory (radial vs. cartesian), spatial resolution $\left(2.5 \times 2.5 \times 2.5 \mathrm{~mm}^{3}\right.$ vs. $\left.3 \times 3 \times 3 \mathrm{~mm}^{3}\right)$, number of cardiac frames (20 vs. 40$)$, and scan duration $(9-14 \mathrm{~min}$. vs. $16-57 \mathrm{~min}$.), although it is unclear how this difference led to the observed differences in flow compartment distribution. Despite the fact that our baseline LV flow compartment distribution in controls differs from that in the literature, our finding of flow compartment shifts in AMI patients relative to controls is valid since all of the $4 \mathrm{D}$ flow data presented herein was acquired with the same sequence.

Peak systolic flow in the LV base was negatively correlated with heart rate and circumferential strain and positively correlated with stroke volume and ejection fraction. The negative correlation of peak flow with heart rate may result from compensatory heart rate increases in order to maintain cardiac output in the infarcted individuals with reduced intraventricular flow. The negative correlation with circumferential strain means that individuals with the largest magnitude of strain had the highest flow in the LV base (because circumferential strain is negative). Ventricles that deformed more where able to push more blood through at peak systole. This correlation may not have been significant in other LV regions due to sample size limitations. The positive correlations of peak systolic intraventricular flows with SV and EF are expected since SV is the total flow over one cardiac cycle at the aortic valve, and EF is directly related to SV. The lack of significant correlation between the LV flow metrics and infarct size could be attributed to the small sample size of the MI group. 

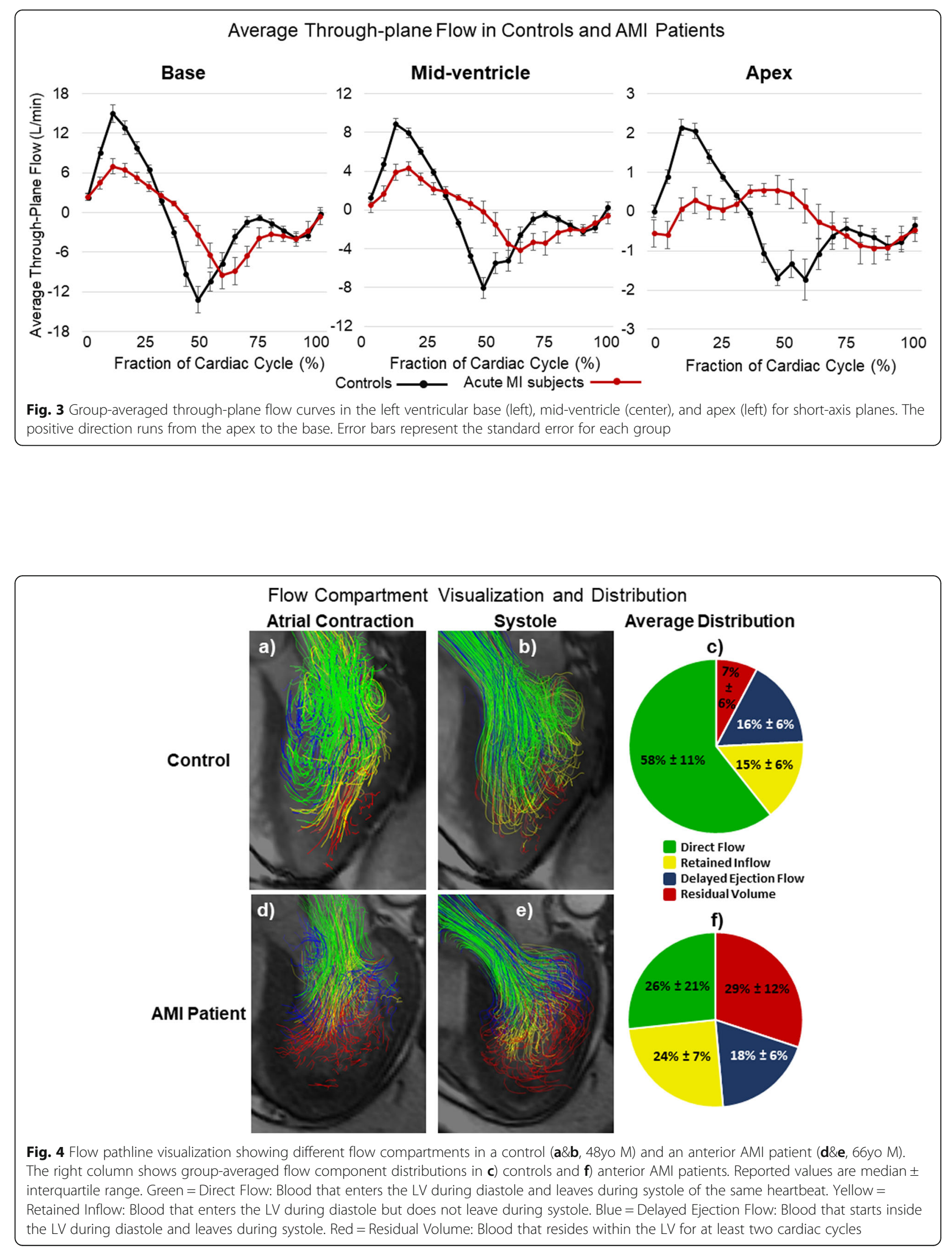
Table 3 LV flow parameters in controls and anterior AMI patients

\begin{tabular}{|c|c|c|c|c|c|c|}
\hline Flow Parameter & Region & Controls $(n=19)$ & Anterior AMI $(n=12)$ & $P$-value & $\Delta_{\mathrm{Ml}}$ & $95 \% \mathrm{Cl}$ \\
\hline \multirow[t]{3}{*}{ Peak Systolic Flow (L/min) } & Base & $16.2 \pm 5.4$ & $8.4 \pm 6.8$ & 0.28 & -2.1 & {$[-5.8,1.6]$} \\
\hline & Mid-ventricle & $8.5 \pm 3.6$ & $5.5 \pm 3$ & 0.01 & -3.9 & {$[-6.4,-1.4]$} \\
\hline & Apex & $2.2 \pm 1$ & $1.4 \pm 1.4$ & 0.02 & -1.4 & {$[-2.6,-0.3]$} \\
\hline \multirow[t]{3}{*}{ Peak Diastolic Flow (L/min) } & Base & $18.6 \pm 6.9$ & $13.3 \pm 4.7$ & 0.71 & 1.1 & {$[-4.6,6.8]$} \\
\hline & Mid-ventricle & $12 \pm 4.3$ & $9.1 \pm 4.3$ & 0.35 & -1.6 & {$[-4.9,1.7]$} \\
\hline & Apex & $3.1 \pm 1.5$ & $2 \pm 1.1$ & 0.004 & -2.4 & {$[-4.0,-0.9]$} \\
\hline Average $\mathrm{KEi}_{\text {EDV }}(\mu \mathrm{J} / \mathrm{mL})$ & Whole LV & $11.3 \pm 2.7$ & $16.6 \pm 11.9$ & 0.79 & -0.9 & {$[-8.0,6.1]$} \\
\hline Direct Flow \% & Whole LV & $58 \pm 11$ & $26 \pm 21$ & $4 \mathrm{E}-08$ & -41 & {$[-52,-31]$} \\
\hline Retained Inflow \% & Whole LV & $15 \pm 6$ & $24 \pm 7$ & .0003 & 12 & {$[7,18]$} \\
\hline Delayed Ejection Flow \% & Whole LV & $16 \pm 6$ & $18 \pm 6$ & 0.005 & 8 & {$[3,13]$} \\
\hline Residual Volume \% & Whole LV & $7 \pm 6$ & $29 \pm 12$ & $6 \mathrm{E}-06$ & 21 & {$[14,28]$} \\
\hline
\end{tabular}

Note-Reported values are median \pm interquartile range (IQR). $P$-values were computed using multivariable linear regression models with age, sex, and heart rate as covariates. Bold text represents a significant $P$-value. $\Delta_{\mathrm{Ml}}$ is the expected difference in the LV flow parameter due to the anterior AMI. $95 \% \mathrm{Cl}$ is the $95 \%$ confidence interval for $\Delta_{\mathrm{MI}}$

$\mathrm{KEi}_{\mathrm{EDV}}$ : Kinetic energy indexed to end diastolic volume

\section{Limiations}

One limitation of this pilot study is the relatively low number of subjects in each cohort. Additionally, 4D flow data in patients was collected as an adjunct to clinical CMR exams. Accordingly, multiple scanners (1.5 T and $3 \mathrm{~T}$ ) were used depending on clinical availability. As a result, imaging parameters and image quality slightly varied between patients, and quality might have been compromised in comparison to controls (all imaged at 3 $\mathrm{T})$. The use of $1.5 \mathrm{~T}$ scanners for some AMI patients limited sensitivity but did not create a systematic bias in AMI patient flow versus controls, as data from Lotz et al. show that the use of $1.5 \mathrm{~T}$ versus $3 \mathrm{~T}$ scanners reduces flow measurement precision but not accuracy [25]. Likewise, the difference in contrast agent used between the two groups may have created different blood $\mathrm{T} 1$ constants and therefore velocity-to-noise ratios in the two groups but would not create a bias between the through-plane flow measurements in each group.
The use of different scanners and contrast agents may have affected our KE measurements, however, as erroneously measured high-velocity voxels can dominate the KE sum since it is derived from velocity squared. Moreover, the velocity encoding parameter selection at 100$150 \mathrm{~cm} / \mathrm{s}$ may have been higher than optimal for detecting differences in low velocities (stasis), such as those present in the apex. However, a lower velocity encoding parameter would have resulted in aliased and incorrect flow measurements in the LV base and mid-ventricle. Further studies investigating and validating the optimal 4D flow acquisition settings in the context of AMI are warranted. While the relatively low sample size, increased noise in patient data, and perhaps suboptimal VENC setting may have reduced the power of this study, this study did shed light on which regions of the intraLV flow field are most affected after anterior AMI. Another limitation of this study is the comparison of the prospectively acquired AMI cohort with the control

Table 4 P-values for correlation of regional LV flow parameters with traditional CMR parameters in anterior AMI patients

\begin{tabular}{|c|c|c|c|c|c|c|}
\hline & \multicolumn{3}{|c|}{ Peak Systolic Flow (L/min) } & \multicolumn{3}{|c|}{ Peak Diastolic Flow (L/min) } \\
\hline & Base & Mid-ventricle & Apex & Base & Mid-ventricle & Apex \\
\hline Infarct size & 0.81 & 0.19 & 0.66 & 0.36 & 0.14 & 0.3 \\
\hline Heart rate & $0.02(-0.64)$ & 0.14 & 0.28 & 0.85 & 0.13 & 0.17 \\
\hline Stroke volume & $0.03(0.63)$ & 0.11 & 0.28 & 0.72 & 0.57 & 0.35 \\
\hline Cardiac output & 0.46 & 0.99 & 0.22 & 0.59 & 0.77 & 0.83 \\
\hline Ejection fraction & $0.001(0.84)$ & $0.03(0.62)$ & 0.19 & 0.38 & 0.79 & 0.24 \\
\hline End diastolic volume & 0.97 & 0.84 & 0.23 & 0.9 & 0.56 & 0.66 \\
\hline Global peak longitudinal strain & 0.13 & 0.35 & 0.67 & 0.8 & 0.57 & 0.33 \\
\hline Regional peak radial strain & 0.08 & 0.2 & 0.6 & 0.67 & 0.96 & 0.42 \\
\hline Regional peak circumferential strain & $0.02(-0.69)$ & 0.15 & 0.67 & 0.65 & 0.92 & 0.43 \\
\hline
\end{tabular}

Note-Shown are P-values for spearman's correlation coefficient. For P-values $<0.05$, the correlation coefficient is included in parentheses, and the box is in boldface text. Through-plane flow and strain were compared on a region-by-region basis (i.e. flow in the LV base was compared with strain in the LV base) 
cohort from a previous study. As such, there were differences between the AMI and control groups such as age, sex and heart rate. While our model corrected for differences in age, sex, and heart rate between the groups, we acknowledge that a prospectively matched study would provide stronger evidence of post-AMI flow reductions.

\section{Conclusions}

This study provided a methodology for the regional analysis of 4D LV flow data and applied that methodology in anterior AMI patients and healthy controls. AMI subjects demonstrated reduced through-plane flow in the LV, even after correcting for age, sex, and heart rate differences. Further investigation is necessary to determine whether regional LV flow has predictive value in the post-AMI population.

\section{Abbreviations}

2D: two-dimensional; 3D: three-dimensional; 4D flow: Four-dimensional flow; AMI: Acute myocardial infarction; bSSFP: balanced steady-state free precession; CMR: Cardiovascular magnetic resonance; ECG: Electrocardiogram; FA: Flip angle; FOV: Field of view; LGE: Late gadolinium enhancement; LV: Left ventricle; LVEF: Left ventricular ejection fraction; LVT: Left ventricular thrombus; MI: Myocardial infarction; SNR: Signal-to-noise Ratio; TE: Echo time; TR: Repetition time; VNR: Velocity-to-noise Ratio

\section{Acknowledgements}

The authors gratefully acknowledge Donald G. Benson for assisting CJF with scoring LGE images for infarct size, and Geng Li for providing statistics support.

\section{Authors' contributions}

PAC performed the analysis and prepared the manuscript. OW and JWW conceived of the initial idea and design for the study. JAM developed initial tools for 3-segment compartmental analysis of LV flow. NRA recruited patients and assisted with study design. CJF performed LGE image scoring and contributed to the study design. All authors read and approved the final manuscript.

\section{Funding}

The project was supported by the Clinical and Translational Science Award (CTSA) program, through the NIH National Center for Advancing Translational Sciences (NCATS), grants UL1TR002373 and TL1TR002375. JWW is supported by NIH grant number 1R01HL128278-01. The content is solely the responsibility of the authors and does not necessarily represent the official views of the $\mathrm{NIH}$. The $\mathrm{NIH}$ had no role in the study design and data collection, analysis, interpretation of data, or writing of the manuscript. GE Healthcare provides research support to the University of Wisconsin-Madison. University of Wisconsin-Madison's Radiology Department provided funding for human subjects and MR scanner time for volunteers.

\section{Availability of data and materials}

The datasets analyzed during the current study are available from the corresponding author on reasonable request.

\section{Ethics approval and consent to participate}

This study was approved by the University of Wisconsin-Madison Health Sciences Institutional Review Board (Title: CE MRI Using Investigational Devices, Reference \#: 2016-1347) and was compliant with the Health Insurance Portability and Accountability Act. In accordance with our IRB protocol, written informed consent was obtained from all subjects.

\section{Consent for publication}

Not applicable because all images shown are entirely unidentifiable and there are no details on individuals reported within the manuscript.

\section{Competing interests}

The authors declare that they have no competing interests.

\section{Author details}

'Department of Medical Physics, University of Wisconsin-Madison, 1111 Highland Ave, Madison, WI 53705, USA. ${ }^{2}$ Department of Radiology, University of Wisconsin-Madison, 600 Highland Ave, Madison, WI 53792, USA.

${ }^{3}$ Department of Medicine, University of Wisconsin-Madison, 600 Highland Ave, Madison, WI 53792, USA. ${ }^{4}$ Departments of Medicine and Radiology, Weill Cornell Medical College, 520 East 70th Street, Starr Pavilion, 4th Floor, New York, NY 10021, USA. 5 Departments of Medical Physics and Radiology, University of Wisconsin-Madison, 1111 Highland Ave, Madison, WI 53705, USA.

Received: 20 June 2019 Accepted: 18 December 2019 Published online: 30 December 2019

\section{References}

1. Ward MJ, Kripalani S, Zhu Y, et al. Incidence of emergency department visits for ST-elevation myocardial infarction in a recent six-year period in the United States. Am J Cardiol. 2015;115:167-70. https://doi.org/10.1016/j. amjcard.2014.10.020.

2. McManus DD, Gore J, Yarzebski J, et al. Recent trends in the incidence, treatment, and outcomes of patients with STEMI and NSTEMI. Am J Med. 2011;124:40-7. https://doi.org/10.1016/j.amjmed.2010.07.023.

3. Hombach V, Grebe O, Merkle N, et al. Sequelae of acute myocardial infarction regarding cardiac structure and function and their prognostic significance as assessed by magnetic resonance imaging. Eur Heart J. 2005; 26:549-57. https://doi.org/10.1093/eurheartj/ehi147.

4. Sacco RL, Adams R, Albers G, et al. Guidelines for prevention of stroke in patients with ischemic stroke or transient ischemic attack: a statement for healthcare professionals from the American Heart Association/American Stroke Association Council on stroke - cosponsored by the council on. Circulation. 2006;113:409-49. https://doi. org/10.1161/01.STR.0000199147.30016.74

5. Weinsaft JW, Kim J, Medicherla CB, et al. A novel imaging algorithm for post myocardial infarction left ventricular Thrombus - regional LV function on echocardiography as a gatekeeper for Thrombus evaluation by delayed enhancement cardiac magnetic resonance. JACC Cardiovasc Imaging. 2016; 9:505-15. https://doi.org/10.1016/j.jcmg.2015.06.017.A.

6. Delewi R, Zijlstra F, Piek JJ. Left ventricular thrombus formation after acute myocardial infarction. Heart. 2012;98:1743-9. https://doi.org/10.1136/ heartjnl-2012-301962.

7. Van Dantzig JM, Delemarre BJ, Bot H, et al. Doppler left ventricular flow pattern versus conventional predictors of left ventricular thrombus after acute myocardial infarction. J Am Coll Cardiol. 1995;25:1341-6. https://doi. org/10.1016/0735-1097(94)00548-5

8. Markl M, Frydrychowicz A, Kozerke S, et al. 4D flow MRI. J Magn Reson Imaging. 2012;36:1015-36. https://doi.org/10.1002/jmri.23632.

9. Dyverfeldt $\mathrm{P}$, Bissell M, Barker AJ, et al. 4D flow cardiovascular magnetic resonance consensus statement. J Cardiovasc Magn Reson. 2015;17:72. https://doi.org/10.1186/s12968-015-0174-5.

10. Garg P, Crandon S, Swoboda PP, et al. Left ventricular blood flow kinetic energy after myocardial infarction - insights from 4D flow cardiovascular magnetic resonance. J Cardiovasc Magn Reson. 2018;201(20):61. https://doi. org/10.1186/s12968-018-0483-6.

11. Frydrychowicz A, Wieben O, Niespodzany E, et al. Quantification of thoracic blood flow using volumetric magnetic resonance imaging with radial velocity encoding: in vivo validation. Investig Radiol. 2013;48:819-25. https:// doi.org/10.1097/RLI.0b013e31829a4f2f.

12. Gu T, Korosec FR, Block WF, et al. PC VIPR: a high-speed 3D phase-contrast method for flow quantification and high-resolution angiography. Am J Neuroradiol. 2005;26:743-9 https://doi.org/26/4/743[pii].

13. Johnson KM, Lum DP, Turski PA, et al. Improved 3D phase contrast MRI with off-resonance corrected dual echo VIPR. Magn Reson Med. 2008;60:1329-36. https://doi.org/10.1002/mrm.21763.

14. Tufvesson J, Hedstrom E, Steding-Ehrenborg K, et al. Validation and development of a new automatic algorithm for time-resolved segmentation of the left ventricle in magnetic resonance imaging. Biomed Res Int. 2015; 2015:1-12. https://doi.org/10.1155/2015/970357.

15. Heyde $B$, Member $S$, Jasaityte $R$, et al. Elastic image registration versus speckle tracking for 2-D myocardial motion estimation: a direct comparison in vivo. IEEE Trans Image Process. 2013;32:449-59. 
16. Avants BB, Tustison NJ, Stauffer M, et al. The insight ToolKit image registration framework. Front Neuroinform. 2014;8:1-13. https://doi.org/10. 3389/fninf.2014.00044.

17. Gupta V, Bustamante M, Fredriksson A, et al. Improving left ventricular segmentation in four-dimensional flow MRI using intramodality image registration for cardiac blood flow analysis. Magn Reson Med. 2017;00:0-6. https://doi.org/10.1002/mrm.26674.

18. Yushkevich PA, Piven J, Hazlett HC, et al. User-guided 3D active contour segmentation of anatomical structures: significantly improved efficiency and reliability. Neuroimage. 2006;31:1116-28. https://doi.org/10.1016/j. neuroimage.2006.01.015.

19. Eriksson J, Carlhäll C, Dyverfeldt P, et al. Semi-automatic quantification of $4 D$ left ventricular blood flow. J Cardiovasc Magn Reson. 2010;12:9. https://doi. org/10.1186/1532-429X-12-9.

20. Kim RJ, Wu E, Rafael A, et al. The use of contrast-enhanced magnetic resonance imaging to identify reversible myocardial dysfunction. N Engl J Med. 2000;343:1445-53. https://doi.org/10.1056/NEJM200011163432003.

21. Kim WY, Walker PG, Pedersen EM, et al. Left ventricular blood flow patterns in normal subjects: a quantitative analysis by three-dimensional magnetic resonance velocity mapping. J Am Coll Cardiol. 1995;26:224-38 10.1016/ 0735-1097(95)00141-L.

22. Kanski M, Arvidsson PM, Töger J, et al. Left ventricular fluid kinetic energy time curves in heart failure from cardiovascular magnetic resonance 4D flow data. J Cardiovasc Magn Reson. 2015;17:1-10. https://doi.org/10.1186/ S12968-015-0211-4.

23. Wong J, Chabiniok R, DeVecchi A, et al. Age-related changes in intraventricular kinetic energy: a physiological or pathological adaptation? Am J Physiol Heart Circ Physiol. 2016;310:H747-55. https://doi.org/10.1152/ ajpheart.00075.2015

24. Eriksson J, Bolger AF, Ebbers T, Carlhäll CJ. Four-dimensional blood flowspecific markers of LV dysfunction in dilated cardiomyopathy. Eur Heart J Cardiovasc Imaging. 2013;14:417-24. https://doi.org/10.1093/ehjci/jes159.

25. Lotz J, Döker R, Noeske $R$, et al. In vitro validation of phase-contrast flow measurements at $3 \mathrm{~T}$ in comparison to 1.5 T: precision, accuracy, and signalto-noise ratios. J Magn Reson Imaging. 2005;21:604-10. https://doi.org/10. 1002/jmri.20275

\section{Publisher's Note}

Springer Nature remains neutral with regard to jurisdictional claims in published maps and institutional affiliations.

Ready to submit your research? Choose BMC and benefit from:

- fast, convenient online submission

- thorough peer review by experienced researchers in your field

- rapid publication on acceptance

- support for research data, including large and complex data types

- gold Open Access which fosters wider collaboration and increased citations

- maximum visibility for your research: over $100 \mathrm{M}$ website views per year

At $\mathrm{BMC}$, research is always in progress.

Learn more biomedcentral.com/submissions 